\title{
PREVALENCIA DE TRATAMIENTOS DE PULPOTOMÍA Y PULPECTOMÍA EN LAS CLÍNICAS INTEGRALES DEL NIÑO EN LA UNIVERSIDAD SANTO TOMÁS ENTRE 2007 A 2011
}

\author{
${ }^{1}$ Xiomara Jimena Noriega Herrera, ${ }^{2}$ Martha Juliana Rodríguez Gómez, \\ ${ }^{3}$ Claudia Cáceres Rodríguez \\ ${ }^{1}$ Estudiante X semestre F. de Odontología U. Santo Tomás, Colombia. \\ ${ }^{2}$ Odontóloga U. Javeriana, Especialista en Odontopediatría y Ortodoncia Preventiva U. CES, Candidata Magíster en Epidemiología U. Industrial de Santander, \\ Docente U. Santo Tomás, Colombia. ${ }^{3}$ Odontóloga U. Santo Tomás, Docente U. Santo Tomás Colombia.
}

Autor responsable de correspondencia: Claudia Cáceres Rodríguez

Correo electrónico: claudiacaceres22@yahoo.com

\begin{abstract}
RESUMEN
Objetivo: determinar la prevalencia de tratamientos de pulpotomía y pulpectomía registrados en las historias clínicas de las Clínicas Integrales del Niño I, II y III de la Facultad de Odontología de la Universidad Santo Tomás en Bucaramanga, en el periodo entre 2007 a 2011. Materiales y métodos: se realizó un estudio retrospectivo de corte transversal, se evaluaron 3396 Historias Clínicas (HC) y se incluyeron aquellas con registros de tratamientos pulpares (pulpotomía, pulpectomía) en dientes deciduos verificados con la radiografía final. Entre las variables analizadas estaban la edad, género, municipio de residencia, diagnóstico relacionado con el tratamiento y año de realización. Se obtuvieron proporciones para las variables cualitativas, y medidas de tendencia central y dispersión para las cuantitativas. En el análisis bivariado se realizó la prueba de $\mathrm{Chi}^{2}$ o test Exacto de Fisher. Se considero un valor de $\mathrm{p} \leq \mathrm{a} 0,05$ como estadísticamente significativo.

Resultados: se obtuvieron 2697 HC de las 3396. De estas, 751 (27,8\%) tenían registrado algún tipo de tratamiento pulpar, sin embargo, sólo 425 (56,6\%) presentaban radiografia final. Seiscientos cuarenta y nueve dientes presentaron terapia pulpar, 497 (76,6\%) presentaron pulpotomía y $152(23,4 \%)$ pulpectomía; el molar con mayor número de tratamientos fue el primer molar inferior con 233 (63,6\%) tratamientos.

Conclusión: la prevalencia de HC con radiografia final de tratamiento en las Clínicas Integrales del Niño I, II y III fue $16,1 \%$ en el periodo entre 2007 al 2011. La caries dental fue el diagnóstico más registrado previo al tratamiento pulpar. En el año 2009 se observó un aumento en la realización de tratamientos pulpares. [Noriega XJ, Rodríguez MJ, Cáceres C. Prevalencia de tratamientos de pulpotomía y pulpectomía en las Clínica Integrales del Niño en la Universidad Santo Tomás entre 2007 a 2011. Ustasalud 2013; 12: 33 - 40]
\end{abstract}

Palabras clave: Prevalencia, Pulpotomía, Pulpectomía, Caries dental, Dientes deciduos.

\section{PREVALENCE OF PULPOTOMY AND PULPECTOMY TREATMENTS IN CLÍNICAS INTEGRALES DEL NIÑO AT SANTO TOMAS UNIVERSITY BETWEEN 2007 - 2011}

\section{ABSTRACT}

Objective: to establish the prevalence of pulpotomy and pulpectomy treatments recorded in the medical records of the Clínicas Integrales del Niño I, II and III of the Faculty of Dentistry at Santo Tomas University in Bucaramanga, in the period from 2007 to 2011.

Methods: a retrospective cross-sectional study was done with 3396 dental records. Those records with pulp treatments (pulpotomy, pulpectomy) in primary teeth with the final radiograph were included. Among the variables analyzed were type of pulp treatment, age, gender, county of residence, treatment-related diagnostic and year of completion. Proportions for qualitative variables, means and dispersion measures for quantitative ones were obtained. In bivariate analysis $\mathrm{Chi}^{2}$ test or Fisher exact test was performed. P value less than 0.05 was considered statistically significant.

Results: 2697 dental records were evaluated because 669 were not available. Of these, 751 (27.8\%) had registered some type of pulp treatment, however, only 425 (56.6\%) had final radiograph. Six hundred forty-nine teeth had pulp therapy, 497 (76.6\%) had pulpotomy and $152(23.4 \%)$ pulpectomy, the first lower molar was the one with the highest number of treatments with $233(63.6 \%)$.

Conclusion: the prevalence of pulpar treatment in primary dentition in the Clínicas Integrales del Niño I, II and III was $16.1 \%$ in the period 2007 to 2011. Dental caries was the more frequent diagnosis related to pulp treatment. In 2009, it was an increase in the performance of pulp treatments.

Key words: Prevalence, Pulpotomy, Endodontic treatment, Dental caries, Primary teeth. 


\section{INTRODUCCIÓN}

La dentición primaria requiere especial atención por parte de los padres o adultos que se encuentren al cuidado de los niños puesto que cualquier alteración que ocurra en ella puede generar secuelas en la dentición permanente. Tales cuidados incluyen cepillar y usar la seda dental en el niño, evitar los hábitos nócivos y asistir a controles odontológicos regularmente, entre otros. ${ }^{1} \mathrm{El}$ manejo del comportamiento de los infantes en la consulta odontológica puede llegar a ser complicado, especialmente en los casos en los que se requiere eliminar el dolor como al realizar exodoncias o tratamientos pulpares. ${ }^{2-4}$

Las patologías pulpares en niños se consideran un problema de salud pública debido a las posibles consecuencias que estos eventos ocasionan como inasistencia a clases, disminución en la calidad de vida, alteración en el sistema estomatognático, entre otros. ${ }^{3}$ Adicionalmente, la causa de pérdida de dientes en la dentición decidua se relaciona principalmente con la presencia de caries dental con compromiso pulpar y en segundo lugar con las lesiones traumáticas de los tejidos dentales. ${ }^{5}$

La prevalencia de los problemas pulpares son comunes en la dentición decidua de acuerdo con los datos registrados en algunos servicios de odontopediatría. Por ejemplo, en la Universidad Nacional del Nordeste (UNNE), en Argentina, aproximadamente el $50 \%$ de los tratamientos realizados en niños son terapias pulpares. ${ }^{5}$ Así mismo, en el Servicio de Odontopediatría del Instituto Autónomo Hospital Universitario de los Andes en Mérida (Venezuela), las infecciones periapicales en dientes primarios se presentan en un $27,3 \%$ del total de 908 historias clínicas evaluadas, siendo el primer molar inferior el diente más afectado $(50,8 \%){ }^{6}$

El tratamiento de la patología pulpar en dientes primarios puede ser exitoso siempre y cuando se realice un buen diagnóstico, se haga una técnica adecuada y se usen los materiales apropiados; de esta manera, los dientes permaneceran como unidades funcionales en el arco hasta su exfoliación. Vij y colaboradores (2004) revisaron 226 registros de molares deciduos a los que se les había realizado terapia pulpar vital, ya fuera Tratamiento Pulpar Indirecto (TPI) o pulpotomia con formocresol, encontraron que el TPI fue exitoso en un $94 \%$ de los casos mientras que la pulpotomia lo fue en el $70 \%{ }^{7}$

Si bien en el ámbito internacional se encuentran pocos estudios sobre la prevalencia de tratamientos pulpares en dentición temporal, en Colombia no se encuentran reportes publicados sobre el tema. Por lo tanto, el objetivo del presente trabajo fue determinar la prevalencia de tratamientos de pulpotomía y pulpectomía registrados en las historias clínicas de las Clínicas Integrales del Niño I, II y III de la Universidad Santo Tomás en Bucaramanga, en el periodo de 2007 a 2011.

\section{MATERIALES Y MÉTODOS}

Se realizó un estudio retrospectivo de corte transversal. La muestra de estudio estuvo conformada por el número total (3396) de historias clínicas pertenecientes a las Clínicas Integrales del Niño I, II y III de la Universidad Santo Tomás, Bucaramanga durante el periodo de 2007 a 2011, según el dato obtenido por el Sistema de Información de Procedimientos de las Clínicas Odontológicas (SIPCO).

Se seleccionaron e incluyeron en el estudio las historias clínicas de odontopediatría con registros de tratamientos pulpares (pulpotomía y pulpectomía) en dientes deciduos que pudieran ser certificados con la radiografia final del tratamiento y se excluyeron las historias cuya radiografía final de tratamiento pulpar estuviese en malas condiciones de tal manera que no permitiera observar con facilidad la terapia realizada.

Como variables de salida se tuvieron en cuenta los tratamientos pulpares (pulpotomía y pulpectomía) realizados. Para este estudio, se considero a la pulpotomía como el procedimiento en el que se remueve la pulpa cameral de los molares deciduos verificable mediante su registro en la historia clínica y la presencia de radio-opacidad en la cámara. De otro lado, la pulpectomía fue la remoción de todo el tejido pulpar (cameral y radicular) reportado en la historia clínica y sustentado por la radiografia en la que se observó radio-opacidad en los conductos radiculares. Las variables explicatorias fueron la edad, el género, municipio en el que residía el menor, año de realización del tratamiento, diagnóstico pulpar asociado con el tratamiento y diente tratado.

\section{Procedimiento}

La información fue recolectada por uno de los investigador es (XJN) mediante un instrumento en el que se registraron las variables descritas. Se solicitó el listado completo de las historias clínicas correspondientes a las Clínicas Integrales del Niño I, II y III durante el período 2007 a 2011.

Se realizó una prueba piloto con el 1\% (34) de las historias clínicas que conformaban la totalidad de la muestra; éstas fueron seleccionadas aleatoriamente mediante la función de números aleatorios de Excel. ${ }^{8}$ Posteriormente, se solicitó el permiso a la Dirección de las Clínicas de la Facultad de Odontología para revisar cada una de las 3396 historias; 
se tramitó el acceso a la información a través del área de Administración y Archivo de la Universidad Santo Tomás. Los datos fueron registrados en el instrumento ya mencionado, éste fue diligenciado en cada ocasión de acuerdo con la información obtenida a partir de las historias clínicas incluidas.

La recolección de la información se realizó en la oficina de Archivo Pasivo de la Universidad Santo Tomás en Floridablanca a partir del 11 de febrero hasta el 9 de marzo de 2013. Para la revisión de la información se usaron todas las medidas de bioseguridad necesarias (guantes, tapabocas y gorro).

La información se digitó por duplicado en una base de datos creada para tal fin en Excel. Las dos bases fueron validadas mediante el software Epidata 3,1 para detectar errores de digitación que fueron corregidos hasta obtener una base completamente depurada. Ésta se exportó al paquete estadístico Stata I/C versión 12,0 para su análisis. . $^{8-10}$

\section{Análisis estadístico}

Se realizó un análisis descriptivo de acuerdo con la naturaleza de cada variable, para las variables cualitativas se utilizaron frecuencias y proporciones; para las variables cuantitativas se utilizaron las medidas de tendencia central y dispersión. En el caso del análisis bivariado, se realizó una prueba de $\mathrm{Chi}^{2}$ o test exacto de Fisher entre el género y las variables registradas en la historia clínica. Con este análisis se consiguió establecer la relación entre las variables. Un valor de $\mathrm{p} \leq 0,05$ fue considerado significativo.

\section{Consideraciones éticas}

Según el Artículo 11 del Capítulo I, de los Aspectos Éticos de la Investigación en Seres Humanos, Titulo II de la Resolución No 008430 de 1993 del Ministerio de Salud de la República de Colombia, este trabajo se clasificó como una investigación sin riesgo. Estos estudios "emplean técnicas y métodos de investigación documental restrospectivos y aquellos en los que no se realiza ninguna intervención o modificación intencionada de las variables biológicas, fisiológicas, sicológicas o sociales de los individuos que participan en el estudio, entre los que se consideran: revisión de historias clínicas, entrevistas, cuestionarios y otros en los que no se le identifique ni se traten aspectos sensitivos de su conducta."11 Adicionalmente, se respetaron los principios de confiabilidad y veracidad en el manejo de la información.

\section{RESULTADOS}

El total de historias de odontopediatría de las Clínicas Integrales del Niño I, II y III correspondiente a los años 2007 a 2011 fue 3396. De éstas, 699 no estaban disponibles en archivo por pérdida o porque la historia se podía encontrar en uso. De tal manera que se revisaron 2697 historias clínicas. La proporción de respuesta fue de $79,4 \%$.

De las 2697 historias disponibles, 1946 (72,2\%) no presentaban tratamiento pulpar y $751(27,8 \%)$ sí. Cuatrocientos veinticinco $(56,6 \%)$ presentaban tratamiento pulpar con radiografía final y $326(43,4 \%)$ no incluían la radiografia que certificará la terapia endodóntica aunque si estaba registrado que se había hecho (Figura 1).

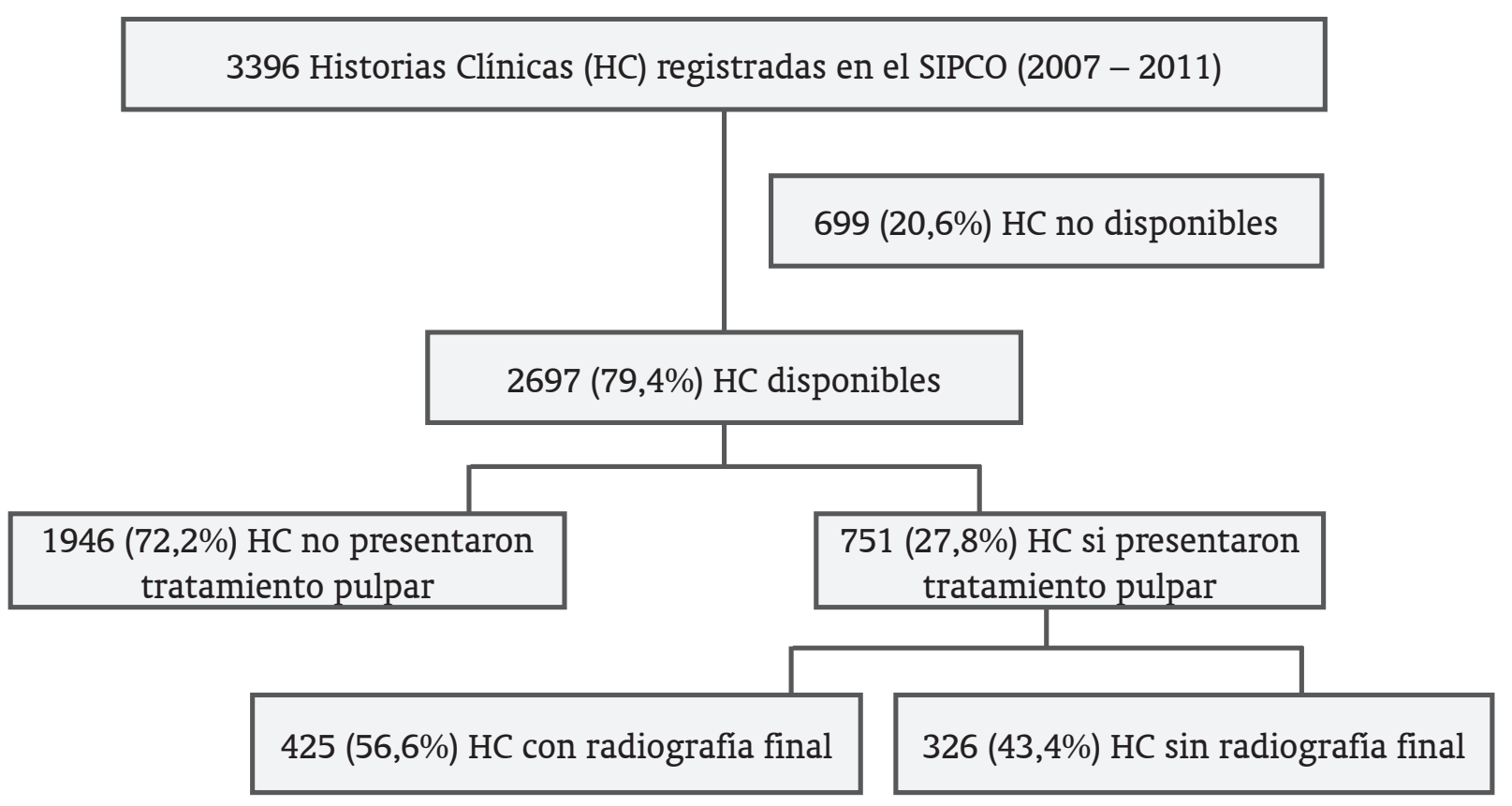

Figura 1. Diagrama de flujo de las historias de las Clínicas Integrales del Niño I, II y III revisadas 


\section{ARTÍCULO DE INVESTIGACIÓN CIENTÍFICA Y TECNOLÓGICA}

De las 425 historias que cumplían con los criterios de inclusión, ocho presentaron tratamientos pulpares realizados en años diferentes pero en el mismo paciente. Por tal motivo, éstas ocho se contaron como si fueran nuevas historias clínicas, de tal manera que el resultado final de historias en las que se pudo verificar un tratamiento pulpar realizado fue 433. Es importante mencionar que 77 historias clínicas presentaban más de un tratamiento pulpar pero algunos de estos procedimientos no incluían la radiografía final por lo que no fueron tenidos en cuenta.
El promedio de edad de los niños con tratamientos pulpares en dientes deciduos fue 6,2 $\pm 1,6$ años con un mínimo de 3 años y un máximo de 11 . Doscientos cincuenta y un $(58,0 \%)$ pacientes eran de género masculino y 245 (56,6\%) vivían en Bucaramanga. El año en que más historias clínicas tenían registrados tratamientos pulpares fue 2010 con 115 (26,6\%) tratamientos (Tabla 1). Ciento seis historias presentaban más de un tratamiento de pulpotomía y 24 reportaron más de un tratamiento de pulpectomía (Tabla 2).

Tabla 1. Características sociodemográficas de las historias clínicas evaluadas según el género

\begin{tabular}{|c|c|c|c|}
\hline Variable & $\begin{array}{c}\text { Femenino } \\
\text { n (\%) }\end{array}$ & $\begin{array}{c}\text { Masculino } \\
\text { n (\%) }\end{array}$ & $\mathbf{P}^{\mathrm{a}}$ \\
\hline Edad & & & 0,470 \\
\hline 3 a 5 años & $63(40,4)$ & $93(59,6)$ & \\
\hline 6 a 8 años & $107(44,2)$ & $135(55,8)$ & \\
\hline 9 a 11 años & $12(34,3)$ & $23(65,7)$ & \\
\hline Municipio de residencia & & & 0,734 \\
\hline Bucaramanga & $110(44,9)$ & $135(55,1)$ & \\
\hline Floridablanca & $30(37,0)$ & $51(63,0)$ & \\
\hline Girón & $10(40,0)$ & $15(60,0)$ & \\
\hline Piedecuesta & $17(39,6)$ & $26(60,4)$ & \\
\hline Otros & $15(38,5)$ & $24(62,5)$ & \\
\hline Año de realizado el tratamiento & & & 0,289 \\
\hline 2007 & $24(42,1)$ & $33(57,9)$ & \\
\hline 2008 & $23(57,5)$ & $17(42,5)$ & \\
\hline 2009 & $49(43,0)$ & $65(57,0)$ & \\
\hline 2010 & $44(38,3)$ & $71(62,7)$ & \\
\hline 2011 & $42(39,3)$ & $65(60,7)$ & \\
\hline Presenta pulpotomía & & & 0,989 \\
\hline No & $32(42,1)$ & $44(57,9)$ & \\
\hline $\mathrm{Si}$ & $150(42,0)$ & $207(58,0)$ & \\
\hline Presenta pulpectomía & & & 0,535 \\
\hline No & $134(43,0)$ & $178(57,0)$ & \\
\hline $\mathrm{Si}$ & $48(39,7)$ & $73(60,3)$ & \\
\hline
\end{tabular}

a: $\mathrm{Chi}^{2}$.

Tabla 2. Número de tratamientos de pulpotomía y de pulpectomía en cada una de las historias clínicas evaluadas según el género

\begin{tabular}{|c|c|c|c|}
\hline Variable & $\begin{array}{c}\text { Femenino } \\
\text { n (\%) }\end{array}$ & $\begin{array}{c}\text { Masculino } \\
\text { n (\%) }\end{array}$ & $\mathbf{P}^{\mathbf{a}}$ \\
\hline Número de pulpotomías por historia & & & 0,737 \\
\hline Una & $102(40,6)$ & $149(59,4)$ & \\
\hline Dos & $36(43,9)$ & $46(56,1)$ & \\
\hline Tres & $9(56,3)$ & $7(43,7)$ & \\
\hline Cuatro & $2(33,3)$ & $4(66,7)$ & \\
\hline Cinco & $1(50,0)$ & $1(50,0)$ & \\
\hline Número de pulpectomías por historia & & & 0,738 \\
\hline Una & $38(39,2)$ & $59(60,8)$ & \\
\hline Dos & $9(47,4)$ & $10(52,6)$ & \\
\hline Tres & $1(33,3)$ & $2(66,7)$ & \\
\hline Cuatro & -- & $2(100,0)$ & \\
\hline
\end{tabular}

a: Test Exacto de Fisher. 
Se observaron 649 dientes tratados con pulpotomía o pulpectomía en el periodo de 2007 a 2011; de estos, $497(76,6 \%)$ presentaban pulpotomía y $152(23,4 \%)$ pulpectomía. De los 497 dientes tratados con pulpotomía, $324(65,2 \%)$ correspondieron a molares inferiores y de los 152 dientes con pulpectomía, 78 (71,5\%) se localizaban en el maxilar superior.

$\mathrm{Al}$ relacionar el género con el diente tratado, se observó que se realizaron 283 (56,9\%) pulpoto- mías en el género masculino. Se encontraron 197 $(39,6 \%)$ pulpotomías en el primer molar inferior en ambos géneros (Tabla 3). De la misma manera, se evidenciaron $93(61,2 \%)$ pulpectomías en el género masculino y el primer molar inferior fue el diente con mayor número de pulpectomías con 36 (23,7\%) tratamientos, le siguió el segundo molar inferior con $31(20,4 \%)$ y el incisivo central superior con 24 $(15,8 \%)$ (Tabla 4).

Tabla 3. Frecuencia de pulpotomías de acuerdo con el diente tratado y el género

\begin{tabular}{|c|c|c|c|c|}
\hline \multirow[b]{2}{*}{ Variable } & \multirow[b]{2}{*}{$\begin{array}{l}\text { Total } \\
\text { n (\%) }\end{array}$} & \multicolumn{2}{|c|}{ Pulpotomía } & \multirow[b]{2}{*}{$\mathbf{P}^{\mathbf{a}}$} \\
\hline & & $\begin{array}{c}\text { Femenino } \\
\mathbf{n}(\%)\end{array}$ & $\begin{array}{c}\text { Masculino } \\
\text { n (\%) }\end{array}$ & \\
\hline Diente tratado & & & & 0,153 \\
\hline \multicolumn{5}{|l|}{ Arcada superior } \\
\hline Primer molar derecho & $53(10,7)$ & $26(49,1)$ & $27(50,9)$ & \\
\hline Segundo molar derecho & $36(7,2)$ & $15(41,7)$ & $21(58,3)$ & \\
\hline Primer molar izquierdo & $45(9,1)$ & $22(48,9)$ & $23(51,1)$ & \\
\hline Segundo molar izquierdo & $39(7,8)$ & $14(35,9)$ & $25(64,1)$ & \\
\hline \multicolumn{5}{|l|}{ Arcada inferior } \\
\hline Primer molar izquierdo & $90(18,1)$ & $37(41,1)$ & $53(58,9)$ & \\
\hline Segundo molar izquierdo & $64(12,9)$ & $34(53,1)$ & $30(46,9)$ & \\
\hline Primer molar derecho & $107(21,5)$ & $35(32,7)$ & $72(67,3)$ & \\
\hline Segundo molar derecho & $63(12,7)$ & $31(49,2)$ & $32(50,8)$ & \\
\hline Total & $497(100,0)$ & $214(43,1)$ & $283(56,9)$ & \\
\hline
\end{tabular}

a: $\mathrm{Chi}^{2}$.

Tabla 4. Frecuencia de pulpectomías de acuerdo con el género y el diente tratado

\begin{tabular}{|c|c|c|c|c|}
\hline \multirow[b]{2}{*}{ Variable } & \multirow[b]{2}{*}{$\begin{array}{l}\text { Total } \\
\text { n (\%) }\end{array}$} & \multicolumn{2}{|c|}{ Pulpectomía } & \multirow[b]{2}{*}{$\mathbf{P}^{\mathbf{a}}$} \\
\hline & & $\begin{array}{c}\text { Femenino } \\
\text { n (\%) }\end{array}$ & $\begin{array}{c}\text { Masculino } \\
\text { n (\%) }\end{array}$ & \\
\hline Diente tratado & & & & 0,814 \\
\hline \multicolumn{5}{|l|}{ Arcada superior } \\
\hline Incisivo central derecho & $13(8,6)$ & $4(30,8)$ & $9(69,2)$ & \\
\hline Incisivo lateral derecho & $6(4,0)$ & $2(33,3)$ & $4(66,7)$ & \\
\hline Canino derecho & $6(4,0)$ & $1(16,7)$ & $5(83,3)$ & \\
\hline Primer molar derecho & $6(4,0)$ & $1(16,7)$ & $5(83,3)$ & \\
\hline Segundo molar derecho & $12(7,9)$ & $6(50,0)$ & $6(50,0)$ & \\
\hline Incisivo central izquierdo & $11(7,2)$ & $4(36,4)$ & $7(63,6)$ & \\
\hline Incisivo lateral izquierdo & $7(4,6)$ & $3(42,9)$ & $4(57,1)$ & \\
\hline Primer molar izquierdo & $5(3,3)$ & $3(60,0)$ & $2(40,0)$ & \\
\hline Segundo molar izquierdo & $12(7,9)$ & $4(33,3)$ & $8(66,7)$ & \\
\hline \multicolumn{5}{|l|}{ Arcada inferior } \\
\hline Canino izquierdo & $5(3,3)$ & $2(40,0)$ & $3(60,0)$ & \\
\hline Primer molar izquierdo & $17(11,2)$ & $5(29,4)$ & $12(70,6)$ & \\
\hline Segundo molar izquierdo & $18(11,8)$ & $11(61,1)$ & $7(38,9)$ & \\
\hline Canino derecho & $2(1,3)$ & $1(50,0)$ & $1(50,0)$ & \\
\hline Primer molar derecho & $19(12,5)$ & $7(36,8)$ & $12(63,2)$ & \\
\hline Segundo molar derecho & $13(8,4)$ & $5(38,5)$ & $8(61,6)$ & \\
\hline Total & $152(100,00)$ & $59(38,8)$ & $93(61,2)$ & \\
\hline
\end{tabular}


El mayor número de tratamientos pulpares se realizó entre los 6 y los 8 años en el género masculino y femenino. Al relacionar el tipo de tratamiento con el año según el género, se observó que el año en que se realizaron más tratamientos de pulpotomía fue el
2009 en el que se registraron 138 (27,8\%) tratamientos. Con respecto a la pulpectomía, se encontró que en el 2011 se realizaron $53(34,9 \%)$ tratamientos y en el año 2008, se hicieron $10(6,6 \%)$ tanto en niñas como en niños (Tabla 5) (Figuras 2 y 3).

Tabla 5. Frecuencia de pulpotomías y pulpectomías según el género, la edad y el año de realizado el tratamiento

\begin{tabular}{|c|c|c|c|c|c|c|}
\hline \multirow[b]{2}{*}{ Variable } & \multicolumn{2}{|c|}{ Pulpotomía } & \multirow[b]{2}{*}{$\mathbf{P}^{\mathbf{a}}$} & \multicolumn{2}{|c|}{ Pulpectomia } & \multirow[b]{2}{*}{$\mathbf{P}^{\mathrm{b}}$} \\
\hline & $\begin{array}{c}\text { Femenino } \\
\text { n (\%) }\end{array}$ & $\begin{array}{c}\text { Masculino } \\
\text { n (\%) }\end{array}$ & & $\begin{array}{c}\text { Femenino } \\
\mathbf{n}(\%)\end{array}$ & $\begin{array}{c}\text { Masculino } \\
\text { n (\%) }\end{array}$ & \\
\hline Edad & & & 0,117 & & & 0,837 \\
\hline 3 a 5 años & $69(38,8)$ & $109(61,3)$ & & $26(37,1)$ & $44(62,9)$ & \\
\hline 6 a 8 años & $134(46,9)$ & $152(53,2)$ & & $30(41,1)$ & $43(58,9)$ & \\
\hline 9 a 11 años & $11(33,3)$ & $22(66,7)$ & & $3(33,3)$ & $6(66,7)$ & \\
\hline \multicolumn{2}{|c|}{ Año de realizado el tratamiento } & & 0,246 & & & 0,375 \\
\hline 2007 & $22(38,6)$ & $35(61,4)$ & & $7(43,8)$ & $9(56,2)$ & \\
\hline 2008 & $26(57,8)$ & $19(42,2)$ & & $6(60,0)$ & $4(40,0)$ & \\
\hline 2009 & $62(45,0)$ & $76(55,0)$ & & $9(28,1)$ & $23(71,9)$ & \\
\hline 2010 & $55(41,4)$ & $78(58,6)$ & & $18(43,9)$ & $23(56,1)$ & \\
\hline 2011 & $49(39,5)$ & $75(60,5)$ & & $19(35,9)$ & $34(64,1)$ & \\
\hline
\end{tabular}

a: $\mathrm{Chi}^{2}$. b: Test Exacto de Fisher.

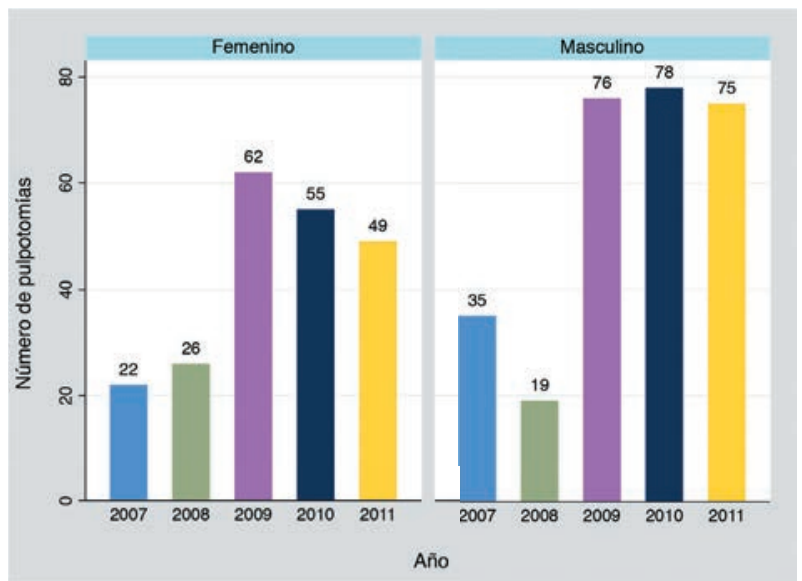

Figura 2. Frecuencia de pulpotomías realizadas según el año y el género.

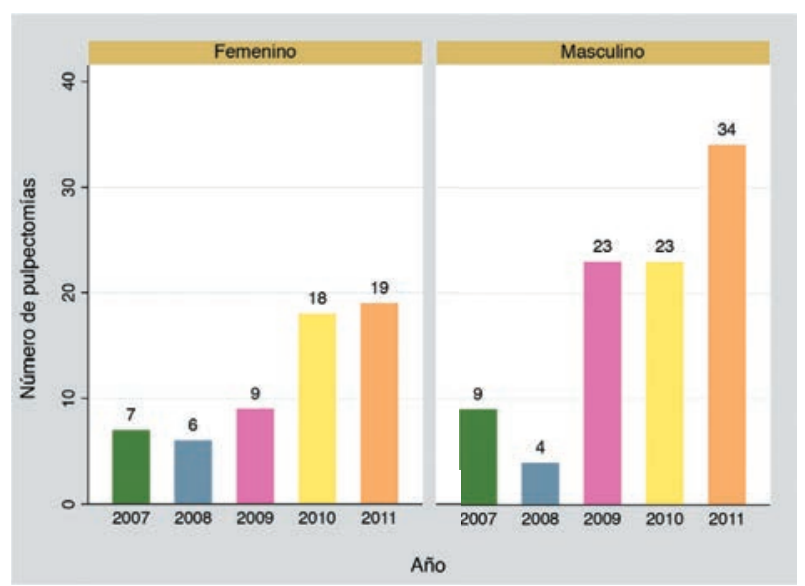

Figura 3. Frecuencia de pulpectomías realizadas según el año y el género.
En relación con el diagnóstico que apoyó el tratamiento realizado, se encontró que $234(47,1 \%)$ correspondieron a caries dental mientras que 192 $(38,6 \%)$ fueron pulpitis reversible. En $16(3,2 \%)$ historias clínicas no se mencionó el diagnóstico por el cual fue tratado el diente con una pulpotomía. De manera similar, $61(40,1 \%)$ diagnósticos de caries dental apoyaron el tratamiento de pulpectomía mientras que $42(27,6 \%)$ pulpectomías se realizaron porque el menor presentó una pulpitis irreversible. Así mismo, se observó una diferencia estadísticamente significativa $(p=0,051)$ entre el género y el diagnóstico registrado para la realización de la pulpotomía (Tabla 6).

\section{DISCUSIÓN}

La prevalencia de historias clínicas con registro de tratamientos pulpares (pulpotomía, pulpectomía) verificados por radiografia final en las Clínicas Integrales del Niño I, II y III fue $16,1 \%$ en el periodo de 2007 a 2011 . Es importante destacar que 326 $(43,4 \%)$ historias clínicas tenían registrados tratamientos pulpares pero éstos no pudieron ser verificados por la ausencia de la radiografía final. En este aspecto es necesario recordar la necesidad de anexar todas las ayudas diagnósticas en la historia clínica respectiva.

El tratamiento pulpar más frecuentemente observado fue la pulpotomía lo que coincide con Fuks quien asegura que la pulpotomía es aún el tratamiento más común. ${ }^{12}$ 
Tabla 6. Frecuencia entre el diagnóstico, el tratamiento realizado y el género

\begin{tabular}{|c|c|c|c|c|c|c|}
\hline \multirow[b]{2}{*}{ Diagnóstico } & \multicolumn{2}{|c|}{ Pulpotomía } & \multirow[b]{2}{*}{$\mathbf{P}^{\mathbf{a}}$} & \multicolumn{2}{|c|}{ Pulpectomía } & \multirow[b]{2}{*}{$\mathbf{P}^{\mathbf{a}}$} \\
\hline & $\begin{array}{c}\text { Femenino } \\
\text { n (\%) }\end{array}$ & $\begin{array}{c}\text { Masculino } \\
\text { n (\%) }\end{array}$ & & $\begin{array}{c}\text { Femenino } \\
\mathrm{n}(\%)\end{array}$ & $\begin{array}{c}\text { Masculino } \\
\text { n (\%) }\end{array}$ & \\
\hline Pulpitis reversible & $96(50,0)$ & $96(50,0)$ & 0,051 & $6(42,9)$ & $8(57,1)$ & 0,850 \\
\hline Pulpitis irreversible & $18(40,9)$ & $26(59,1)$ & & $12(28,6)$ & $30(51,4)$ & \\
\hline Caries dental & $89(38,0)$ & $145(62,0)$ & & $25(41,0)$ & $36(59,0)$ & \\
\hline Necrosis & $0(0,0)$ & $3(100,0)$ & & $6(42,9)$ & $8(57,1)$ & \\
\hline PAAS * & $0(0,0)$ & $0(0,0)$ & & $2(66,7)$ & $1(33,3)$ & \\
\hline PAANS * & $0(0,0)$ & $1(100,0)$ & & $1(50,0)$ & $1(50,0)$ & \\
\hline PACS * & $2(100,0)$ & $0(0,0)$ & & $1(50,0)$ & $1(50,0)$ & \\
\hline PACNS * & $1(20,0)$ & $4(80,0)$ & & $5(45,4)$ & $6(54,6)$ & \\
\hline No menciona & $8(50,0)$ & $8(50,0)$ & & $1(33,3)$ & $2(66,7)$ & \\
\hline Total & $214(43,1)$ & $283(56,9)$ & & $59(38,8)$ & $93(61,2)$ & \\
\hline
\end{tabular}

* PAAS: Periodontitis Apical Aguda No Supurativa. PAANS: Periodontitis Apical Aguda No Supurativa. * PACS: Periodontitis Apical Crónica Supurativa. PACNS: Periodontitis Apical Crónica No Supurativa. a: Test Exacto de Fisher.

De las 649 observaciones de tratamientos pulpares (pulpotomía, pulpectomía) en dientes deciduos encontradas en las 425 historias clínicas que se evaluaron, $376(57,9 \%)$ tratamientos pulpares correspondieron al género masculino. Si bien Barrios y Carrero (2011) estudiaron la prevalencia de la patología periapical, sus hallazgos coinciden con este estudio al observar que $139(56,1 \%)$ niños presentaban esta entidad. ${ }^{6}$ Sin embargo, Martínez y Galiana (2005) encontraron que del total de los niños tratados, el 65,6\% eran niñas y el 34,3\% niños. ${ }^{5}$

A pesar de este hallazgo, no hay una explicación clara del por qué el género masculino puede verse más involucrado en tratamientos pulpares en dentición decidua. Podría sugerirse que a las niñas las cuidan más o que a los niños los llevan más a consulta ya que de las 425 historias clínicas, 248 (58,3\%) pertenecían al género masculino.

La edad en la que más frecuentemente se presentaron tratamientos pulpares fue los 5 y 6 años, 228 $(45,9 \%)$ de los registros en estas edades correspondieron a pulpotomías y $66(43,4 \%)$ a pulpectomías. Los hallazgos de Barrios y Carrero son similares ya que observaron que $173(69,8 \%)$ niños en un rango de edad de 4 a 6 años estaban afectados. ${ }^{6}$

En el periodo de observación (2007 a 2011), se encontró que la prevalencia de pulpotomías y pulpectomías verificadas con radiografía final fue $76,57 \%$ y $23,42 \%$, respectivamente. Estos resultados coinciden con el estudio realizado por Martínez y Galiana al evaluar los niños atendidos en la cátedra de odontopediatría de la Facultad de Odontología de la Universidad Nacional del Noreste, las autoras encontraron que de los 128 tratamientos pulpares realizados, el $81,5 \%$ correspondían a pulpotomías. ${ }^{5}$

En el mismo estudio se observó que los primeros molares inferiores fueron los dientes más afectados. ${ }^{5}$ Este hallazgo coincide con lo registrado en las historias clínicas revisadas puesto que el diente tratado con mayor frecuencia fue también el primer molar inferior. No es de extrañar este resultado ya que en la literatura se ha considerado al primer molar inferior como el molar más susceptible a presentar problemas pulpares debido en parte, a que es el primer molar en erupcionar y por lo tanto, puede ser más vulnerable a caries dental. ${ }^{13}$

Adicionalmente, los primeros molares presentan un espesor dentinal menor en relación con los segundos molares. Al parecer son dientes más permeables y este aspecto es uno de los responsables de la entrada de bacterias e irritantes en la pulpa. ${ }^{14,15}$

En relación con el periodo de tiempo evaluado, se observó que a partir del año 2009, los tratamientos pulpares (pulpotomía, pulpectomía) en los dientes deciduos aumentaron notablemente. Esta situación coincide con el cambio en el sistema de evaluación de las Clínicas Integrales del Niño I, II y III puesto que en este nuevo sistema se tiene en cuenta el tratamiento como procedimiento obligatorio con un porcentaje asignado que influye en la calificación final del estudiante.

De igual manera, se observó que el diagnóstico que más se relacionó con el tratamiento pulpar realizado fue caries dental con $234(47,1 \%)$ en tratamientos de pulpotomías y $61(40,1 \%)$ en pulpectomías. La pulpitis reversible sólo se encontró como razón 
para la realización de la pulpotomía en 192 (38,6\%) casos. Se podría pensar que esta situación se debe a que el estudiante no registra el diagnóstico que en realidad presenta el diente y el docente no lo verifica. También, se podría deducir que se están realizando tratamientos pulpares en dientes con lesiones profundas de caries dental que se encuentran asintomáticos aunque el hecho de presentar una lesión cariosa cavitacional no es indicación de terapia pulpar. $^{16}$

Lo mismo sucedió con los tratamientos de pulpectomía ya que la pulpitis irreversible sólo fue registrada en $42(27,6 \%)$ tratamientos de los 152 realizados. Carrote sugirió que el tratamiento de endodoncia estaba indicado cuando el niño presentaba pulpitis irreversible o necrosis pulpar lo que no se encontró en todos los casos en este estudio. ${ }^{17}$

En cuanto a los diagnósticos, es importante mencionar que algunos no correspondieron teóricamente con el tratamiento realizado, es decir, la literatura sobre terapia pulpar en dentición decidua es clara al especificar cuáles son los diagnósticos que indican determinado tratamiento. ${ }^{18,19}$ Entre los diagnósticos que no corresponden para la realización de una pulpotomía, se encontraron tres $(0,6 \%)$ dientes con necrosis pulpar, uno $(0,2 \%)$ con periodontitis apical aguda no supurativa, cinco $(1,0 \%)$ con periodontitis apical crónica no supurativa y dos $(0,4 \%)$ con periodontitis apical crónica supurativa. Esta misma circunstancia ocurrió con la pulpectomía ya que en 14 $(9,2 \%)$ dientes se registró pulpitis reversible.

Una de las fortalezas de este estudio es la extensa revisión de historias clínicas de odontopediatría. Los resultados obtenidos son una herramienta importante para evaluar el por qué se han incrementado los tratamientos pulpares y son una manera de llamar la atención de estudiantes y docentes para diligenciar y anexar todas las ayudas que soporten los tratamientos realizados en los pacientes que asisten a las Clínicas Integrales del Niño I, II y III.

\section{BIBLIOGRAFÍA}

1. Nahás MS. Odontopedatría en la Primera Infancia. Santos Edictora; 2009.

2. Betancourt M, Fernández M, Valcarcel J. Lesiones pulpares y periapicales en escolares del área de atención del policlínico Docente de Playa. Rev Haban Cienc Med La Habana. 2009; VIII: 1 - 5.

3. Setty JV, Srinivasan I. Awareness and attitude of patients' parents toward pulp therapy of the primary teeth: A clinical survey. J Indian Soc Pedod Prev Dent. 2011; 29: 198 $-201$.

4. Torres EA, Rodríguez MJ, Cañón OL. Manejo del comportamiento del paciente niño en la consulta odontológica. Ustasalud. 2002; 1: 49 - 56.

5. Martinez S, Galiana A. Determinación de la frecuencia de tratamientos pulpares realizados en piezas dentarias primarias en la Cátedra de Odontopediatría de la FOUNNE. Años 2002 - 2003. Universidad Nacional del Nordeste. 2005; URL disponible en: http://www.unne.edu.ar/unnevieja/Web/cyt/com2005/3-Medicina/M-094.pdf.

6. Barrios ZC, Carrero TI. Prevalencia de infecciones de origen pulpar en los niños atendidos en el servicio de odontopediatría del Instituto Autónomo Hospital Universitario de Los Andes, Mérida, Venezuela. Revista Odontológica de Los Andes. 2011; 6: 42 - 51.

7. Vij R, Coll JA, Shelton P, Farooq NS. Caries control and other variables associated with success of primary molar vital pulp therapy. Pediatr Dent. 2004; 26: 214 - 220.

8. Microsoft Corporation. Microsoft Excel. United States: 2010.

9. EpiData Association. Epidata Software 3.1. Denmark: 2004.

10. Stata Statistical Software. Stata Release 12.0. College Station. TX: Stata Corporation: 2011.

11. República de Colombia, Ministerio de Salud. Resolución 008430 de 4 de octubre de 1993.

12. Fuks AB. Vital pulp therapy with new materials for primary teeth: new directions and treatment perspectives. Pediatr Dent. 2008; 30: 211 - 219.

13. Escobar A. Características Clínicas de la Caries Dental. En: Cárdenas D. Odontología Pediátrica. Medellín: Corporación para Investigaciones Biológicas; 2009. p. 131 - 157.

14. Castillhos $\mathrm{H}$, Chevitarese O. A comparative study of dentin thickness of primary human molars. J Clin Pediatr Dent. 2003; 27: 277 - 281.

15. Koutsi V, Nooman RG, Horner JA, Simpson MD, Matthew WG, Pashley DH. The effect of dentin depth on the permeability and ultraestructure of primary molars. Pediatr Dent. 1994; 16: 29 - 35.

16. Fejerskov O, Kidd EAM, Nyvad B, Baelum V. Defining the Disease: An Introduction. En: Fejerskov O, Kidd EAM. Dental Caries: Disease and its Clinical Management. 2008. p. 4 - 6.

17. Carrote P. Endodontic treatment for children. British Dent J. 2005; 198: 10 - 14.

18. Coll JA, Josell S, Nassof S, Shelton P, Richards MA. An evaluation of pulpal therapy in primary incisors. Pediatr Dent. 1988; 10: 178 - 184

19. Rodd H, Waterhouse A, Fuks A, Moffat F. Pulp therapy for primary molars. Int J Paediatr Dent. 2006; 16: 15 - 23. 